\title{
Erratum to: Divisionally free arrangements of hyperplanes
}

\author{
Takuro Abe ${ }^{1}$
}

Published online: 19 December 2016

(C) Springer-Verlag Berlin Heidelberg 2016

\section{Mathematics Subject Classification 32S22 $52 \mathrm{C} 35$}

\section{Erratum to: Invent. math. (2016) 204:317-346 DOI 10.1007/s00222-015-0615-7}

The aim of this note is to correct the statement and the proof of Theorem 6.2 in the paper published in Inventiones Mathematicae 204 (2016), 317-346, which is not correct as it was stated. All the other results in the paper are correct as they were stated. The correct statement of Theorem 6.2 should be as follows:

Theorem 6.2 Let $\mathbb{K}$ be an arbitrary field, $V=\mathbb{K}^{\ell}$ and $\mathcal{A}$ a free arrangement in $V$ with $\exp (\mathcal{A})=\left(1, d_{1}, \ldots, d_{\ell-1}\right)$. Assume that distinct hyperplanes $H_{1}, \ldots, H_{\ell-1} \in \mathcal{A}$ satisfy that,

(1) $\mathcal{A}_{i}^{\prime}:=\mathcal{A} \backslash\left\{H_{i}\right\}$ is free with $\exp \left(\mathcal{A}_{i}^{\prime}\right)=\left(1, d_{1}, d_{2}, \ldots, d_{i-1}, d_{i}-\right.$ $\left.1, d_{i+1}, \ldots, d_{\ell-1}\right)$ for $i=1, \ldots, \ell-1$, and

(2) $\mathcal{A}^{\prime}:=\mathcal{A} \backslash\left\{H_{1}, \ldots, H_{\ell-1}\right\}$ is free with $\exp \left(\mathcal{A}^{\prime}\right)=\left(1, d_{1}-1, \ldots, d_{\ell}-1\right)$.

Then $\mathcal{A} \in \mathcal{D F}$.

Proof The original proof is correct except for the part showing the following statement in its second paragraph; " $D\left(\mathcal{A}^{\prime}\right)$ has a basis $\theta_{E}, \theta_{1}, \ldots, \theta_{\ell-1}$

The online version of the original article can be found under doi:10.1007/s00222-015-0615-7.

Takuro Abe

abe@imi.kyushu-u.ac.jp

1 Institute of Mathematics for Industry, Kyushu University, Fukuoka 819-0395, Japan 
such that the derivations $\theta_{E}, \alpha_{H_{1}} \theta_{1}, \ldots, \alpha_{H_{\ell-1}} \theta_{\ell-1}$ form a basis for $D(\mathcal{A})$ "'. To prove this statement, we have to show that "there is a derivation $\theta_{i} \in$ $D\left(\mathcal{A}_{i}^{\prime}\right)(i=1, \ldots, \ell-1)$ of degree $d_{i}-1$ such that $\theta_{i} \notin D(\mathcal{A})$, but that $\alpha_{H_{i}} \theta_{i} \in D(\mathcal{A})$ ". Since both $\mathcal{A}$ and $\mathcal{A}_{i}^{\prime}$ are free, Terao's addition-deletion theorem implies that there is a basis $\theta_{E}, \theta_{1}^{(i)}, \ldots, \theta_{\ell-1}^{(i)}$ for $D(\mathcal{A})$ such that $\operatorname{deg} \theta_{j}^{(i)}=d_{j}(j=1, \ldots, \ell-1)$ and $\alpha_{H_{i}} \mid \theta_{i}^{(i)}$. If $\theta_{i}:=\theta_{i}^{(i)} / \alpha_{H_{i}}$, then $\theta_{i} \in D\left(\mathcal{A}_{i}^{\prime}\right) \backslash D(\mathcal{A})$. Now the rest proof is the same as the original one.

Except for Theorem 6.2, all the results and proofs are correct in this paper.

Acknowledgements The author is grateful to Torsten Hoge for his pointing out a mistake of the original Theorem 6.2 